\title{
ON THE ACTIVE GRAVITATIONAL MASS OF A NON-SPHERICAL SOURCE LEAVING HYDROSTATIC EQUILIBRIUM
}

\author{
L. Herrera ${ }^{* \dagger}$, A. Di Prisco, E. Fuenmayor ${ }^{\ddagger}$ \\ Escuela de Física, Facultad de Ciencias, \\ Universidad Central de Venezuela, Caracas, Venezuela.
}

November 1, 2018

\begin{abstract}
We obtain an expression for the active gravitational mass (Tolman) of a source of the $\gamma$ metric, just after its departure from hydrostatic equilibrium, on a time scale of the order of (or smaller than) the hydrostatic time scale. It is shown that for very compact sources, even arbitrarily small departures from sphericity, produce significant decreasing (increasing) in the values of active gravitational mass of collapsing (expanding) spheres, with respect to its value in equilibrium, enhancing thereby the stability of the system.
\end{abstract}

*Postal address: Apartado 80793, Caracas 1080A, Venezuela.

†e-mail: laherrera@telcel.net.ve

$\ddagger$ e-mail:efuenma@fisica.ciens.ucv.ve 


\section{Introduction}

One of the most remarkable aspect of general relativity, is the very special status that this theory confers to the spherical symmetry. This situation is particularly well illustrated by the Israel theorem.

Indeed, as is well known [1], the only static and asymptotically-flat vacuum space-time possessing a regular horizon is the Schwarzschild solution. For all the others Weyl exterior solutions [2], the physical components of the Riemann tensor exhibit singularities at $r=2 m$. Therefore, it is intuitively clear that for high gravitational fields, the evolution of sources of Weyl spacetime should drastically differ from the evolution of spherical sources [3]. It is important to keep in mind that the sharp difference in the behaviour of both types of sources (for very high gravitational fields) will exist independently on the magnitude of multipole moments (higher than monopole) of the Weyl source. This is so because, as the source approaches the horizon, any finite perturbation of the Schwarzschild space-time becomes fundamentally different from any Weyl solution, even when the latter is characterized by parameters whose values are arbitrarily close to those corresponding to the spherical symmetry. This point has been stressed before [4, but usually it has been overlooked.

Notwithstanding, spherical symmetry is a common assumption in the study of compact self-gravitating objects (white dwarfs, neutron stars, black holes ), furthermore in the specific case of non-rotating black holes, spherical symmetry should be "absolute", according to Israel theorem. Therefore it is pertinent to ask, how do small deviations from this assumption, related to any kind of perturbation (e.g. fluctuations of the stellar matter, external perturbations, etc), affect the dynamics of the system?.

In a recent work [5] it was shown that for a non-spherical source (even in the case of slight deviations from spherical symmetry), the speed of entering the collapse regime decreases substantially, as compared with the exactly spherically symmetric case. Also, the sensitivity of the trajectories of test particles in the $\gamma$ spacetime, to small changes of $\gamma$, for orbits close to $2 \mathrm{~m}$, has been brought out [6].

It is the purpose of this work to study further the behaviour of axysymmetric sources for very high gravitational fields. This will allow us to put in evidence the role played by the non sphericity (however small) of the source, on the outcome of evolution. To do so we shall obtain an expression for the active gravitational mass of the source, immediately after its departure from 
equilibrium. Here "immediately" means on a time scale of the order of (or smaller than) hydrostatic time scale -see section 4 for more details.

As initial configurations, we shall consider two interior metrics. These ones were found some years ago by Stewart et al. [7, following a prescription given by Hernández [8] allowing to obtain sources of Weyl space time, from known spherically symmetric interior solutions.

The configurations to be considered are sources of the so-called gamma metric ( $\gamma$-metric) 9, 10]. This metric, which is also known as Zipoy-Vorhees metric [11, belongs to the family of Weyl's solutions, and is continuously linked to the Schwarzschild space-time through one of its parameters. The motivation for this choice is twofold. On one hand the exterior $\gamma$-metric corresponds to a solution of the Laplace equation (in cylindrical coordinates) with the same singularity structure as the Schwarzschild solution (a line segment 9]). In this sense the $\gamma$-metric appears as the "natural" generalization of Schwarzschild space-time to the axisymmetric case. On the other hand, the two interiors considered have reasonable physical properties and generalize important and useful sources of the Schwarzschild space-time, namely the interior Schwarzschild solution (homogeneous density) and the Adler solution 12. All this having been said, we would like to emphasize that our main goal here is not to model the behaviour of a specific type of compact object, but to illustrate the effects of slight deviations from spherical symmetry, on the source.

As we shall see, the value of active gravitational mass of the source evaluated just after the departure from equilibrium, will be seriously affected by slight deviations from spherical symmetry.

On the other hand, we are well aware of the fact that the $\gamma$ metric is not the only possible description for the exterior of a compact objetc and, of course, the two equations of state considered here do not exhaust the list of possible candidates for the equation of state of the stellar matter. However, in view of the properties of the $\gamma$ metric and the two equations of state considered here, mentioned above, it is fair to say that the case for the relevance of small deviations from spherical symmetry in the dynamics of compact objects has been further strengthened. This suggests that any conclusion on the structure and evolution of a compact object, derived on the assumption of spherical symmetry should be carefully checked against small deviations from that assumption, whenever the boundary of the source is close to the horizon. 


\section{The Space-time}

\subsection{The exterior space-time}

As it has been mentioned above, our initial matter configuration is the source of an axially symmetric and static space-time ( $\gamma$-metric). In cylindrical coordinates, static axisymmetric solutions to Einstein equations are given by the Weyl metric [2]

$$
d s^{2}=e^{2 \lambda} d t^{2}-e^{-2 \lambda}\left[e^{2 \mu}\left(d \rho^{2}+d z^{2}\right)+\rho^{2} d \varphi^{2}\right],
$$

with

$$
\lambda_{, \rho \rho}+\rho^{-1} \lambda_{, \rho}+\lambda_{, z z}=0
$$

and

$$
\mu_{, \rho}=\rho\left(\lambda_{, \rho}^{2}-\lambda_{, z}^{2}\right) \quad \mu_{, z}=2 \rho \lambda_{, \rho} \lambda_{, z} .
$$

Observe that (2) is just the Laplace equation for $\lambda$ (in the Euclidean space).

The $\gamma$-metric is defined by [9]

$$
\begin{gathered}
\lambda=\frac{\gamma}{2} \ln \left[\frac{R_{1}+R_{2}-2 m}{R_{1}+R_{2}+2 m}\right], \\
e^{2 \mu}=\left[\frac{\left(R_{1}+R_{2}+2 m\right)\left(R_{1}+R_{2}-2 m\right)}{4 R_{1} R_{2}}\right]^{\gamma^{2}},
\end{gathered}
$$

where

$$
R_{1}^{2}=\rho^{2}+(z-m)^{2} \quad R_{2}^{2}=\rho^{2}+(z+m)^{2} .
$$

It is worth noticing that $\lambda$, as given by (4), corresponds to the Newtonian potential of a line segment of mass density $\gamma / 2$ and length $2 m$, symmetrically distributed along the $z$ axis. The particular case $\gamma=1$, corresponds to the Schwarzschild metric.

It will be useful to work in Erez-Rosen coordinates [11, given by

$$
\rho^{2}=\left(r^{2}-2 m r\right) \sin ^{2} \theta \quad z=(r-m) \cos \theta,
$$

which yields the line element as 9

$$
d s^{2}=F d t^{2}-F^{-1}\left\{G d r^{2}+H d \theta^{2}+\left(r^{2}-2 m r\right) \sin ^{2} \theta d \varphi^{2}\right\}
$$


where

$$
\begin{aligned}
F & =\left(1-\frac{2 m}{r}\right)^{\gamma}, \\
G & =\left(\frac{r^{2}-2 m r}{r^{2}-2 m r+m^{2} \sin ^{2} \theta}\right)^{\gamma^{2}-1},
\end{aligned}
$$

and

$$
H=\frac{\left(r^{2}-2 m r\right)^{\gamma^{2}}}{\left(r^{2}-2 m r+m^{2} \sin ^{2} \theta\right)^{\gamma^{2}-1}}
$$

Now, it is easy to check that $\gamma=1$ corresponds to the Schwarzschild metric.

The total mass of the source is [9, 10] $M=\gamma m$, and the quadrupole moment is given by

$$
Q=\frac{\gamma}{3} m^{3}\left(1-\gamma^{2}\right)
$$

So that $\gamma>1(\gamma<1)$ corresponds to an oblate (prolate) spheroid.

\subsection{The interior space-time}

The metric within the matter distribution bounded by the surface

$$
r=r_{\Sigma}
$$

is given by

$$
\begin{aligned}
g_{t t} & =f^{2 \gamma} \\
g_{r r} & =-f^{2(1-\gamma)} \Delta^{\gamma^{2}-2} \Sigma^{1-\gamma^{2}} \\
g_{\theta \theta} & =-r^{2} f^{2 \gamma(\gamma-1)} \Phi^{1-\gamma^{2}} \\
g_{\varphi \varphi} & =-r^{2} f^{2(1-\gamma)} \sin ^{2} \theta
\end{aligned}
$$

where $f, \Delta, \Sigma$ and $\Phi$ are functions whose specific form depends on the model under consideration.

The two cases to be considered here are reported in [7, namely

1. The modified constant density Schwarzschild solution

$$
f(r)=\frac{3}{2} \sqrt{1-\frac{r_{\Sigma}^{2}}{B^{2}}}-\frac{1}{2} \sqrt{1-\frac{r^{2}}{B^{2}}}
$$




$$
\begin{aligned}
\Delta(r) & =1-\frac{r^{2}}{B^{2}} \\
\Sigma(r, \theta) & =1-\frac{r^{2}}{B^{2}}+\frac{r^{4}}{4 B^{4}} \sin ^{2} \theta \\
\Phi(r, \theta) & =f^{2}+\frac{r^{4}}{4 B^{4}} V(r) \sin ^{2} \theta
\end{aligned}
$$

with

$$
V(r)=1+\frac{6}{r}_{\Sigma}\left(r_{\Sigma}-r\right),
$$

and

$$
B^{2}=\frac{3}{8 \pi \rho_{s s}},
$$

where $\rho_{s s}$ denotes the energy density in the spherically symmetric limit $(\gamma=1)$

2. The modified Adler solution

$$
\begin{aligned}
f(r) & =A+B r^{2} \\
\Delta(r) & =1+\frac{C r^{2}}{\left(A+3 B r^{2}\right)^{2 / 3}} \\
\Sigma(r, \theta) & =1+\frac{C r^{2}}{\left(A+3 B r^{2}\right)^{2 / 3}}+\frac{C^{2} r^{4}}{4\left(A+3 B r^{2}\right)^{4 / 3}} \sin ^{2} \theta \\
\Phi(r, \theta) & =\left(A+B r^{2}\right)^{2}+\frac{C^{2} r^{4} V(r)}{4\left(A+3 B r^{2}\right)^{4 / 3}} \sin ^{2} \theta
\end{aligned}
$$

with

$$
V(r)=1+\frac{6}{r_{\Sigma}}\left(1-\frac{5 m}{3 r_{\Sigma}}\right)\left(1-\frac{m}{r_{\Sigma}}\right)^{-1}\left(r_{\Sigma}-r\right)
$$

and

$$
A=\frac{1-\frac{5 m}{2 r_{\Sigma}}}{\left(1-\frac{2 m}{r_{\Sigma}}\right)^{1 / 2}}
$$




$$
\begin{aligned}
B & =\frac{m}{2 r_{\Sigma}^{3}\left(1-\frac{2 m}{r_{\Sigma}}\right)^{1 / 2}} \\
C & =-\frac{2 m\left(1-\frac{m}{r_{\Sigma}}\right)^{2 / 3}}{r_{\Sigma}^{3}\left(1-\frac{2 m}{r_{\Sigma}}\right)^{1 / 3}}
\end{aligned}
$$

Before closing this section, two remarks are in order:

1. Since we are considering the source described in (14) as an initial state, the time derivatives of functions $f, \Delta, \Sigma$ and $\Phi$ will be in principle different from zero.

2. Junction (Darmois) conditions are satisfied at the boundary $r=r_{\Sigma}$ -see [] for details.

\section{The energy momentum tensor}

In order to give physical meaning to the components of the energy momentum tensor in coordinates $(t, r, \theta, \varphi)$, we shall develop a procedure similar to that used by Bondi [13] in his study of non static spherically symmetric sources. Thus, we introduce purely local Minkowski coordinates $(\tau, x, y$,

$z$ ) (alternatively one may introduce a tetrad field associated with locally Minkowskian observers) defined by

$$
\begin{aligned}
& d \tau=f^{\gamma} d t \\
& d x=f^{1-\gamma} \Delta^{-1+\gamma^{2} / 2} \Sigma^{\left(1-\gamma^{2}\right) / 2} d r \\
& d y=r f^{\gamma(\gamma-1)} \Phi^{\left(1-\gamma^{2}\right) / 2} d \theta \\
& d z=r \sin (\theta) f^{1-\gamma} d \varphi .
\end{aligned}
$$

Next, since we are assuming that our source does not dissipate energy, then the covariant components of the energy momentum tensor, as measured 
by a local Minkowskian and comoving with the fluid observer, will be

$$
\widehat{T}_{\mu \nu}=\left(\begin{array}{cccc}
\rho & 0 & 0 & 0 \\
0 & p_{x x} & p_{x y} & 0 \\
0 & p_{y x} & p_{y y} & 0 \\
0 & 0 & 0 & p_{z z}
\end{array}\right)
$$

where $\rho$ is the energy density and in general $p_{x x} \neq p_{y y} \neq p_{z z}$ and $p_{x y}=p_{y x}$. We may write (32) in the form

$\widehat{T}_{\mu \nu}=\left(\rho+p_{z z}\right) \widehat{U}_{\mu} \widehat{U}_{\nu}-p_{z z} \eta_{\mu \nu}+\left(p_{x x}-p_{z z}\right) \widehat{k}_{\mu} \widehat{k}_{\nu}+\left(p_{y y}-p_{z z}\right) \widehat{l}_{\mu} \widehat{l}_{\nu}+2 p_{x y} \widehat{k}_{(\mu} \widehat{l}_{\nu)}$,

where $\eta_{\mu \nu}$ denotes the flat space-time metric and

$$
\begin{aligned}
\widehat{U}_{\mu} & =\left(\begin{array}{llll}
1, & 0, & 0, & 0
\end{array}\right) \\
\widehat{k}_{\mu} & =\left(\begin{array}{llll}
0, & 1, & 0, & 0
\end{array}\right) \\
\widehat{l}_{\mu} & =\left(\begin{array}{llll}
0, & 0, & 1, & 0
\end{array}\right)
\end{aligned}
$$

The components of the energy-momentum tensor $T_{\mu \nu}$ in $(t, r, \theta, \varphi)$ coordinates are linked to (33) by

$$
T_{\mu \nu}=\Lambda_{\mu}^{\alpha} \Lambda_{\nu}^{\beta} L_{\alpha}^{\gamma} L_{\beta}^{\delta} \widehat{T}_{\gamma \delta},
$$

where the non vanishing components of $\Lambda_{\mu}^{\nu}$ are

$$
\begin{aligned}
\Lambda_{t}^{\tau} & =f^{\gamma} \\
\Lambda_{r}^{x} & =f^{1-\gamma} \Delta^{-1+\gamma^{2} / 2} \Sigma^{\left(1-\gamma^{2}\right) / 2} \\
\Lambda_{\theta}^{y} & =r f^{\gamma(\gamma-1)} \Phi^{\left(1-\gamma^{2}\right) / 2} \\
\Lambda_{\varphi}^{z} & =r \sin (\theta) f^{1-\gamma} .
\end{aligned}
$$

and the Lorentz matrices $L_{\mu}^{\nu}$ are given by

$$
L_{t}^{t}=\Gamma \quad L_{i}^{t}=L_{t}^{i}=-\Gamma w_{i} \quad L_{j}^{i}=L_{i}^{j}=\delta_{j}^{i}+\frac{(\Gamma-1) w_{i} w_{j}}{w^{2}}
$$


where

$$
w^{2}=w_{x}^{2}+w_{y}^{2} \quad \Gamma=\frac{1}{\sqrt{1-w^{2}}},
$$

and $w_{x}$ and $w_{y}$ denote, respectively, the velocity of a fluid element along the $x$ and $y(r$ and $\theta)$ directions, as measured by our local Minkowskian observer as defined by (28)-(31). Observe that we are considering the case $w_{z}=0$, which means that the system preserves the reflection symmetry (besides the axial symmetry).

Then, (37) readily gives

$$
\begin{aligned}
T_{t t}= & f^{2 \gamma} \Gamma^{2}\left(\rho+p_{x x} w_{x}^{2}+p_{y y} w_{y}^{2}+2 p_{x y} w_{x} w_{y}\right) \\
T_{t r}= & -f \Delta^{-1+\gamma^{2} / 2} \Sigma^{\left(1-\gamma^{2}\right) / 2} \Gamma \times \\
& \left(\Gamma w_{x} \rho+p_{x x} w_{x} \Lambda_{x}+p_{y y} w_{y} \Lambda+p_{x y}\left[w_{x} \Lambda+w_{y} \Lambda_{x}\right]\right) \\
T_{t \theta}= & -r f^{\gamma^{2}} \Phi^{\left(1-\gamma^{2}\right) / 2} \Gamma \times \\
& \left(\Gamma w_{y} \rho+p_{x x} w_{x} \Lambda+p_{y y} w_{y} \Lambda_{y}+p_{x y}\left[w_{y} \Lambda+w_{x} \Lambda_{y}\right]\right) \\
T_{r r}= & f^{2-2 \gamma} \Delta^{\gamma^{2}-2} \Sigma^{1-\gamma^{2}} \times \\
& \left(\Gamma^{2} w_{x}^{2} \rho+p_{x x} \Lambda_{x}^{2}+p_{y y} \Lambda^{2}+2 p_{x y} \Lambda \Lambda_{x}\right) \\
T_{r \theta}= & r f^{(\gamma-1)^{2}} \Delta^{-1+\gamma^{2} / 2} \Phi^{\left(1-\gamma^{2}\right) / 2} \Sigma^{\left(1-\gamma^{2}\right) / 2} \times \\
& \left(\Gamma^{2} w_{x} w_{y} \rho+\Lambda^{2}\left[p_{x x} \Lambda_{x}+p_{y y} \Lambda_{y}\right]+p_{x y}\left[\Lambda^{2}+\Lambda_{x} \Lambda_{y}\right]\right) \\
T_{\theta \theta}= & r^{2} f^{2 \gamma(\gamma-1)} \Phi^{1-\gamma^{2}} \times \\
& \left(\Gamma^{2} w_{y}^{2} \rho+p_{x x} \Lambda^{2}+p_{y y} \Lambda_{y}^{2}+2 p_{x y} \Lambda \Lambda_{y}\right) \\
T_{\varphi \varphi}= & r^{2} f^{2(1-\gamma)} \sin ^{2}(\theta) p_{z z}, \\
& \\
& \\
= &
\end{aligned}
$$

with

$$
\begin{aligned}
\Lambda & \equiv \frac{(\Gamma-1) w_{x} w_{y}}{w^{2}} \\
\Lambda_{x} & \equiv 1+\frac{(\Gamma-1) w_{x}^{2}}{w^{2}}
\end{aligned}
$$




$$
\Lambda_{y} \equiv 1+\frac{(\Gamma-1) w_{y}^{2}}{w^{2}}
$$

So that

$T_{\mu \nu}=\left(\rho+p_{z z}\right) U_{\mu} U_{\nu}-p_{z z} g_{\mu \nu}+\left(p_{x x}-p_{z z}\right) k_{\mu} k_{\nu}+\left(p_{y y}-p_{z z}\right) l_{\mu} l_{\nu}+2 p_{x y} k_{(\mu} l_{\nu)}$,

where $U_{\mu}, k_{\mu}$ and $l_{\mu}$ are obtained after applying the boost velocity (42) and (38) - (41) to (34)-(36),

$$
\begin{aligned}
U_{\mu} & =\Gamma\left(\begin{array}{llll}
f^{\gamma}, & -w_{x} f^{1-\gamma} \Delta^{-1+\gamma^{2} / 2} \Sigma^{\left(1-\gamma^{2}\right) / 2}, & -w_{y} r f^{\gamma(\gamma-1)} \Phi^{\left(1-\gamma^{2}\right) / 2}, & 0
\end{array}\right), \\
k_{\mu} & =\left(\begin{array}{llll}
-\Gamma w_{x} f^{\gamma}, & f^{1-\gamma} \Delta^{-1+\gamma^{2} / 2} \Sigma^{\left(1-\gamma^{2}\right) / 2} \Lambda_{x}, & r f^{\gamma(\gamma-1)} \Phi^{\left(1-\gamma^{2}\right) / 2} \Lambda, & 0
\end{array}\right), \\
l_{\mu} & =\left(\begin{array}{llll}
-\Gamma w_{y} f^{\gamma}, & f^{1-\gamma} \Delta^{-1+\gamma^{2} / 2} \Sigma^{\left(1-\gamma^{2}\right) / 2} \Lambda, & r f^{\gamma(\gamma-1)} \Phi^{\left(1-\gamma^{2}\right) / 2} \Lambda_{y}, & 0
\end{array}\right) .
\end{aligned}
$$

In the static case $w_{x}=w_{y}=\Lambda=0$ and $\Gamma=\Lambda_{x}=\Lambda_{y}=1$. The same holds after the system departs from equilibrium, on a time scale of the order of (or smaller than) the hidrostatic time scale (see Section 2.4).

\section{The Tolman mass and departure from equi- librium}

We shall now find an expression for the Tolman mass of the source described in the previous section, an then we shall evaluate it just after its departure from equilibrium, on a time scale of the order of (or smaller than) the hydrostatic time scale. Also, since we are interested in the effects produced by small deviations from spherical symmetry, it will be assumed further that the source is quasi-spherical, and therefore we shall put $\gamma=1+\epsilon$ and will neglect terms of order $O\left(\epsilon^{2}\right)$ or smaller.

\subsection{The Tolman mass}

The Tolman mass is given by [14] 


$$
\begin{aligned}
m_{T} & =\int_{0}^{r_{\Sigma}} \int_{0}^{\pi} \int_{0}^{2 \pi} \sqrt{-g}\left(T_{0}^{0}-T_{1}^{1}-T_{2}^{2}-T_{3}^{3}\right) d r d \theta d \phi \\
& +\frac{1}{8 \pi} \iiint \sqrt{-g} g^{\alpha \beta} \frac{\partial}{\partial t}\left[\frac{\partial \mathcal{L}}{\partial\left(\partial\left(\sqrt{-g} g^{\alpha \beta}\right) / \partial t\right)}\right] d r d \theta d \phi
\end{aligned}
$$

where $\mathcal{L}$ denotes the usual gravitational lagrangian density (eq.(10) in [14]). Although Tolman's formula was introduced as a measure of the total energy of the system, with no commitment to its localization, we shall define the mass for any value of $r$, smaller or equal to $r_{\Sigma}$, as

$$
\begin{aligned}
m_{T} & =\int_{0}^{r} \int_{0}^{\pi} \int_{0}^{2 \pi} \sqrt{-g}\left(T_{0}^{0}-T_{1}^{1}-T_{2}^{2}-T_{3}^{3}\right) d r d \theta d \phi \\
& +\frac{1}{8 \pi} \iiint \sqrt{-g} g^{\alpha \beta} \frac{\partial}{\partial t}\left[\frac{\partial \mathcal{L}}{\partial\left(\partial\left(\sqrt{-g} g^{\alpha \beta}\right) / \partial t\right)}\right] d r d \theta d \phi .
\end{aligned}
$$

This extension of the global concept of energy to a local level [15] is suggested by the conspicuous role played by $m_{T}$ as the "active gravitational mass".

Indeed, it can be easily shown [16] that the gravitational acceleration $a$ of a test particle, instantaneously at rest in a static gravitational field, as measured with standard rods and coordinate clock is given by

$$
a=-\frac{m_{T}}{r^{2}} .
$$

A similar conclusion can be obtained by inspection of the equation of hydrostatic equlibrium (TOV) (in the static or quasi-static, spherically symmetric case case) [17].

Even though these properties of Tolman's definition of mass are only valid in the spherically symmetric and static case, it is reasonable to assume that for small deviations from these conditions, the same role of active gravitational mass may be assigned to our expression.

Let us now evaluate expression (59). After some lengthy calculations one finds:

$$
\begin{aligned}
m_{T}= & 2 \pi \int_{0}^{r} \int_{0}^{\pi} r^{2} \sin \theta f^{\gamma^{2}-2 \gamma+2} \Delta^{\gamma^{2} / 2-1} \Sigma^{\left(1-\gamma^{2}\right) / 2} \Phi^{\left(1-\gamma^{2}\right) / 2} \\
\times & {\left[\Gamma^{2} \rho\left(1+\omega_{x}^{2}+\omega_{y}^{2}\right)+p_{x x}\left(\Gamma^{2} \omega_{x}^{2}+\Lambda_{x}^{2}+\Lambda^{2}\right)+p_{y y}\left(\Gamma^{2} \omega_{y}^{2}+\Lambda_{y}^{2}+\Lambda^{2}\right)\right.} \\
& \left.+p_{z z}+2 p_{x y}\left(\Gamma^{2} \omega_{x} \omega_{y}+\Lambda\left(\Lambda_{x}+\Lambda_{y}\right)\right)\right] d r d \theta
\end{aligned}
$$




$$
\begin{aligned}
& +\quad \frac{1}{4} \int_{0}^{r} \int_{0}^{\pi} r^{2} \sin \theta f^{\gamma^{2}-4 \gamma+2} \Delta^{\gamma^{2} / 2-1} \Sigma^{\left(1-\gamma^{2}\right) / 2} \Phi^{\left(1-\gamma^{2}\right) / 2} \\
& \times \quad\left[2\left(2-3 \gamma+\gamma^{2}\right) \frac{\ddot{f}}{f}+\left(\gamma^{2}-2\right) \frac{\ddot{\Delta}}{\Delta}+\left(1-\gamma^{2}\right) \frac{\ddot{\Phi}}{\Phi}+\left(1-\gamma^{2}\right) \frac{\ddot{\Sigma}}{\Sigma}\right. \\
& + \\
& +2 \gamma\left(1-\gamma^{2}\right)\left(-\gamma^{2}+2 \gamma-3\right)\left(\frac{\dot{f}}{f}\right)^{2}+\frac{1}{2}\left(\gamma^{2}-2\right)\left(\gamma^{2}-4\right)\left(\frac{\dot{\Delta}}{\Delta}\right)^{2} \\
& \quad-\frac{1}{2}\left(1-\gamma^{2}\right)\left(\gamma^{2}+1\right)\left(\left(\frac{\dot{\Phi}}{\Phi}\right)^{2}+\left(\frac{\dot{\Sigma}}{\Sigma}\right)^{2}\right)+\left(\gamma^{2}-2\right)(2-3 \gamma) \frac{\dot{f}}{f} \frac{\dot{\Delta}}{\Delta} \\
& +\left(1-\gamma^{2}\right)(2-3 \gamma) \frac{\dot{f}}{f} \frac{\dot{\Sigma}}{\Sigma}+\gamma\left(1-\gamma^{2}\right)(2 \gamma-3) \frac{\dot{f}}{f} \frac{\dot{\Phi}}{\Phi} \\
& \left.+\left(1-\gamma^{2}\right)\left(\gamma^{2}-2\right) \frac{\dot{\Delta}}{\Delta} \frac{\dot{\Sigma}}{\Sigma}\right] d r d \theta
\end{aligned}
$$

We shall now evaluate this last expression at the very moment the system leaves the equilibrium, and for small non-sphericity.

\subsection{Departure from equilibrium}

Let us now consider that our source, once submitted to perturbations, departs from equilibrium without dissipation. We shall then evaluate the system after such departure, on a time scale such that $w_{x}$ and $w_{y}$ remain vanishingly small, whereas their time derivatives though small, will be different from zero.

Thus, just after leaving the equilibrium, the following conditions hold

$$
\begin{gathered}
w_{x}=w_{y}=w_{x, i}=w_{y, i} \simeq 0, \quad(i=r, \theta, \varphi) \\
\dot{w}_{x}, \dot{w}_{y} \neq 0 \quad \text { (small) }
\end{gathered}
$$

where dots denote derivative with respect to $t$.

From now on, unless otherwise stated, all equations are evaluated at the moment the system starts to deviate from equilibrium.

Then from (44)-(50), we obtain using (62)

$$
T_{t \theta}=T_{t r}=0
$$

which implies, because of field equations (see [5] for details)

$$
\dot{\Delta}=\dot{f}=\dot{\Sigma}=\dot{\Phi}=0
$$


where for simplicity we write 0 for $\mathcal{O}(\omega)$ (as we shall do hereafter).

Obviously, spatial derivatives of the above quantities will be also vanishingly small on the time scale under consideration.

Also, from evaluation of the $t$-component of the conservation law $T_{\nu ; \mu}^{\mu}=0$, we get (see [5]).

$$
\dot{\rho}=0
$$

Then, in the approximation above, the expression for the Tolman mass becomes

$$
\begin{aligned}
m_{T} & =2 \pi \int_{0}^{r} \int_{0}^{\pi} r^{2} \sin \theta f^{\left(\gamma^{2}-2 \gamma+2\right)} \Delta^{\left(\gamma^{2} / 2-1\right)} \Sigma^{\left(1-\gamma^{2}\right) / 2} \Phi^{\left(1-\gamma^{2}\right) / 2} \\
& \times\left(T_{0}^{0}-T_{1}^{1}-T_{2}^{2}-T_{3}^{3}\right) d r d \theta d \phi \\
& +\frac{1}{4} \int_{0}^{r} \int_{0}^{\pi} r^{2} \sin \theta f^{\left(\gamma^{2}-4 \gamma+2\right)} \Delta^{\left(\gamma^{2} / 2-1\right)} \Sigma^{\left(1-\gamma^{2}\right) / 2} \Phi^{\left(1-\gamma^{2}\right) / 2} \\
& \times\left[2\left(\gamma^{2}-3 \gamma+2\right) \frac{\ddot{f}}{f}+\left(\gamma^{2}-2\right) \frac{\ddot{\Delta}}{\Delta}+\left(1-\gamma^{2}\right)\left(\frac{\ddot{\Sigma}}{\Sigma}+\frac{\ddot{\Phi}}{\Phi}\right)\right] d r d \theta d \phi
\end{aligned}
$$

We shall now write $\gamma=1+\epsilon$, then assuming that non-sphericity is small (quasi-spherical approximation), the expression above reads:

$$
\begin{aligned}
m_{T} & =2 \pi \int_{0}^{r} \int_{0}^{\pi} r^{2} \sin \theta f \Delta^{(\epsilon-1 / 2)} \Sigma^{-\epsilon} \Phi^{-\epsilon} \times\left(T_{0}^{0}-T_{1}^{1}-T_{2}^{2}-T_{3}^{3}\right) d r d \theta d \phi \\
& +\frac{1}{4} \int_{0}^{r} \int_{0}^{\pi} r^{2} \sin \theta f^{-(1+2 \epsilon)} \Delta^{(\epsilon-1 / 2)} \Sigma^{-\epsilon} \Phi^{-\epsilon} \\
& \times \quad\left[-\frac{\ddot{\Delta}}{\Delta}+2 \epsilon\left(-\frac{\ddot{f}}{f}+\frac{\ddot{\Delta}}{\Delta}-\frac{\ddot{\Sigma}}{\Sigma}-\frac{\ddot{\Phi}}{\Phi}\right)\right] d r d \theta d \phi
\end{aligned}
$$

Within our two approximations (quasi-spherical and (62)-(65)) it follows from (44)-(53) that:

$$
T_{0}^{0}-T_{1}^{1}-T_{2}^{2}-T_{3}^{3}=\rho+p_{x x}+p_{y y}+p_{z z}
$$

Also within these approximations, the time derivative of the $\operatorname{tr}$ component of the Einstein equations $G_{r t}=-8 \pi T_{r t}$, yields

$$
\begin{aligned}
& -\frac{1}{r} \frac{\ddot{\Delta}}{\Delta}+2 \epsilon\left[\frac{\ddot{\Phi}^{\prime}}{2 \Phi}+\frac{\Phi^{\prime}}{2 \Phi} \frac{\ddot{\Delta}}{2 \Delta}+\frac{1}{r}\left(\frac{\ddot{\Delta}}{\Delta}-\frac{\ddot{\Sigma}}{\Sigma}-\frac{\ddot{f}}{f}+\frac{\ddot{\Phi}}{2 \Phi}\right)-\frac{\ddot{\Phi}}{2 \Phi}\left(\frac{f^{\prime}}{f}+\frac{\Phi^{\prime}}{\Phi}\right)\right] \\
& =8 \pi f \Delta^{(\epsilon-1 / 2)} \Sigma^{-\epsilon}\left[\dot{\omega}_{x}\left(\rho+p_{x x}\right)+\dot{\omega}_{y} p_{x y}\right] .
\end{aligned}
$$


Feeding back (69) and (170) into (68), one obtains after some rearrangements

$$
\begin{aligned}
m_{T} & =2 \pi \int_{0}^{r} \int_{0}^{\pi} r^{2} \sin \theta f \Delta^{(\epsilon-1 / 2)} \Sigma^{-\epsilon} \Phi^{-\epsilon} \times\left(\rho+p_{x x}+p_{y y}+p_{z z}\right) d r d \theta \\
& +2 \pi \int_{0}^{r} \int_{0}^{\pi} r^{3} \sin \theta f^{-2 \epsilon} \Delta^{(2 \epsilon-1)} \Sigma^{-2 \epsilon} \Phi^{-\epsilon}\left[\dot{\omega}_{x}\left(\rho+p_{x x}\right)+\dot{\omega}_{y} p_{x y}\right] d r d \theta \\
& -\frac{3}{4} \epsilon \iint r^{2} \sin \theta f^{-(1+2 \epsilon)} \Delta^{(\epsilon-1 / 2)} \Sigma^{-\epsilon} \Phi^{-\epsilon} \frac{\ddot{\Phi}}{\Phi} d r d \theta \\
& -\frac{1}{4} \epsilon \iint r^{3} \sin \theta f^{-(1+2 \epsilon)} \Delta^{(\epsilon-1 / 2)} \Sigma^{-\epsilon} \Phi^{-\epsilon} \\
& \times\left[\frac{\ddot{\Phi}^{\prime}}{2 \Phi}+\frac{\Phi^{\prime}}{2 \Phi} \frac{\ddot{\Delta}}{2 \Delta}+\frac{1}{r}\left(\frac{\ddot{\Delta}}{\Delta}-\frac{\ddot{\Sigma}}{\Sigma}-\frac{\ddot{f}}{f}+\frac{\ddot{\Phi}}{2 \Phi}\right)-\frac{\ddot{\Phi}}{2 \Phi}\left(\frac{f^{\prime}}{f}+\frac{\Phi^{\prime}}{\Phi}\right)\right] d r d \theta
\end{aligned}
$$

\subsection{The spherically symmetric case}

Before entering into the discussion of (171), it is quite instructive to analyze

the spherically symmmetric situation. In this case one easily obtains from (171)

$$
\begin{aligned}
m_{T} & =4 \pi \int_{0}^{r} r^{2} f \times\left(\rho+p_{x x}+p_{y y}+p_{z z}\right) d r \\
& +4 \pi \int_{0}^{r} r^{3} \Delta^{-1}\left[\dot{\omega}_{x}\left(\rho+p_{x x}\right)\right] d r
\end{aligned}
$$

with $p_{y y}=p_{z z}$. Now, the first term in (72) correspond to the active gravitational mass of the core of radius $r$ interior to $r_{\Sigma}$ before the system leaves the equilibrium. Let us now assume that the system leaves the equilibrium and starts to collapse (expand), then $\dot{\omega}_{x}<0\left(\dot{\omega}_{x}>0\right)$ decreasing (increasing) the value of the Tolman mass with respect to its value at equilibrium. Thus the second term in (72) tends to stabilize the system, opposing any departure from equilibrium (contraction or expansion). This result was already known [18.

Let us now get back to the non-spherical case to see how small nonsphericities change the whole picture.

\subsection{The non-spherical case}

Let us now turn back to (71), to infer what happens in the non-spherical case (i.e. $\varepsilon \neq 0$ ), even though $\varepsilon<<1$. Then neglecting higher order terms on $\varepsilon$, 
and keeping only the leading terms, we get

$$
\begin{aligned}
m_{T} & =2 \pi \int_{0}^{r} \int_{0}^{\pi} r^{2} \sin \theta f \Delta^{(\epsilon-1 / 2)} \Sigma^{-\epsilon} \Phi^{-\epsilon} \times\left(\rho+p_{x x}+p_{y y}+p_{z z}\right) d r d \theta \\
& +2 \pi \int_{0}^{r} \int_{0}^{\pi} r^{3} \sin \theta f^{-2 \epsilon} \Delta^{(2 \epsilon-1)} \Sigma^{-2 \epsilon} \Phi^{-\epsilon}\left[\dot{\omega}_{x}\left(\rho+p_{x x}\right)\right] d r d \theta
\end{aligned}
$$

In order to extract more information from (73) it is necessary to specify the source under consideration. We shall use the two configurations mentioned in section 2. In both cases, $f$ vanishes at the central region for $r_{\Sigma}>2 m$. Thus, in the Schwarzschild-like models we have $f(0)=0$, if $2 m / r_{\Sigma}=8 / 9$. Since we know that any spherically symmetric static configuration with constant $\rho_{s s}$ and locally isotropic pressure should satisfy the constraint $n \equiv 2 M / r_{\Sigma}<8 / 9$ we may assume that the system leaves the equilibrium for values of $n$ close to 8/9. Then, an inspection of (73) shows that (if $\varepsilon>0$ ), for values of $n$ approaching $8 / 9$, the second term incresases due to the factor $f^{-2 \epsilon}$.

We may now use Einstein equations to elucidate that for negative values of $\varepsilon$ the system may be unphysical. If $n$ takes values close to the limit allowed by the model (8/9 for Schwarzschild-type model and 4/5 for Adlertype model), the critical values $(f \rightarrow 0)$ appear close to $r=0$. Outside of this region the system is basically composed by an spherical incompressible fluid plus a perturbation in $\varepsilon$. Thus, the physical or unphysical character of the model is determined by its behaviour close to these two limits.

The energy density, in the limit $r \rightarrow 0$, for the Schwarzschild-type model and Adler-type model is given by expresions see ([5] for details)

$$
\rho=\frac{m}{2 \pi r_{\Sigma}^{3}} f^{2 \varepsilon}\left[\frac{3}{2}+\varepsilon\left(1+\frac{1}{3 \sqrt{1-\frac{2 m}{r_{\Sigma}}}-1}\right)\right]
$$

and

$\rho=\frac{3 m}{4 \pi r_{\Sigma}^{3}\left(1-2 m / r_{\Sigma}\right)^{1 / 3}}\left(\frac{1-5 m / 2 r_{\Sigma}}{\sqrt{1-2 m / r_{\Sigma}}}\right)^{2 \varepsilon-2 / 3}\left[\left(1-m / r_{\Sigma}\right)^{2 / 3}+\frac{\varepsilon}{\left(1-5 m / 2 r_{\Sigma}\right)^{1 / 3}}\right]$

respectively. From these ones, it is easy to show that if $\varepsilon<0$, the energy density becomes negative as the system approaches to $n \rightarrow 8 / 9$ (Schwarzschild 
case) or $n \rightarrow 4 / 5$ (Adler case) and positive for $\varepsilon \geq 0$. Therefore, in both cases, positive energy conditions impose $\varepsilon \geq 0$, and for very compact objects (close to the limit allowed by the model) the "stabilizying" term in the expression for the active gravitational mass substantially increases in the central region, close to, but before, the maximum allowed value of $n$.

\section{Conclusions}

We have seen so far that (as expected), for high gravitational fields, important differences appear in the way that the spherical and non-spherical systems depart from equilibrium. This conclusion being true even for small nonsphericity.

In the two models considered above, a factor multiplying the stabilizying term in the expression for the active gravitational mass, brings out those differences. Although for the two examples considered here, we have considered $\varepsilon>0$, it is obvious that there exist models with $\varepsilon<0$, in which case the stabilizying term may become very small, leading to highly unstable situations. In fact, it is worth noticing that important differences between the two cases $(\varepsilon>0$ and $\varepsilon<0)$ appear also in the behaviour of the exterior $\gamma$-metric (9], [10])

Thus, on the basis of presented results we may conclude that whatever the model and the sign of $\varepsilon$ would be, any source of Weyl metric would evolve quite differently from the corresponding spherical source, as critical values of $n$ are considered.

In particular, in the examples examined, the departure from equilibrium appears to be affected by a sharp modification in the stabilyzying term of the active gravitational mass, as $n$ approaches its maximum allowed value. This change makes the system more stable, hindering its departure from equilibrium.

Before concluding, the following remarks are in order:

1. Observe that all results above are valid only on a time scale of the order of (or smaller than) hydrostatic time scale. Within this time scale, the general form of the metric and the components of the energymomentum tensor, is the same as in the static case (of course in the former case time dependence of variables has to be taken into account when taking time derivatives of such components) 
2. In [7] it is shown that the two interiors described in Section 2.2, match smoothly (on $r_{\Sigma}$ ) with the $\gamma$ metric, in the static case. That this is also the case after the system departs from equilibrium (on a time scale of the order of, or smaller than, hydrostatic time scale) is apparent from (65) and comments after equation (57) and in the point 1 above. Of course in the fully dynamic case (beyond the hydrostatic time scale), (65) does not longer hold, and matching conditions have to be established. But this is out of the scope of this paper.

\section{acknowledgements}

LH acknowledges financial assistance under grant BFM2000-1322 (M.C.T. Spain).

\section{References}

[1] W. Israel, Phys. Rev. 164, 1776, (1967).

[2] H. Weyl,Ann. Physik, 54, 117 1918, ;ibid 159, 185 (1919); T. LeviCivita,Atti. Accad. Naz. Lincei Rend. Classe Sci.Fis. Mat. Nat. 28, 101 (1919);J. L. Synge, Relativity, the general theory (North-Holland Publ. Co, Amsterdam) (1960).

[3] L. Bel, , Gen. Relativ. Gravitation 1, 337 (1971).

[4] J. Winicour, A.I. Janis and E.T. Newman, Phys. Rev. 176,1507 (1968); A. Janis, E.T Newman and J. Winicour,Phys. Rev. Lett. 20, 878 (1968); F.I. Cooperstock and G.J. Junevicus Nuovo Cimento 16B, 387 (1973).

[5] L. Herrera, A. Di Prisco and J. Martinez, Astr. Space Sci. 277447 (2001).

[6] L. Herrera, F. Paiva and N. O. Santos, Int. J. Modern Phys.D 9649 (2000).

[7] B. Stewart, D. Papadopoulos, L. Witten, R. Berezdivin and L. Herrera, Gen. Rel. Grav., 1497 (1982).

[8] W. Hernández, Phys. Rev., 1531359 (1967). 
[9] F. Espósito and L. Witten ,Phys. Lett. 58B, 357 (1975).

[10] K. S. Virbhadra ,preprint gr-qc/9606004 (1996).

[11] R. Bach and H. Weyl,Math. Z. 13134 (1920); G. Darmois Les equations de la Gravitation Einsteinienne (Gauthier-Villars, Paris) p.36 (1927); G. Erez and N. Rosen, Bull. Res. Council Israel 8F, 47 (1959);D.M Zipoy J. Math. Phys. 7, 1137 (1966);R. Gautreau and J.L. Anderson , Phys.Lett. A 25 , 291 (1967); F.I. Cooperstock and G.J. Junevicus Int. J. Theor. Phys. 9, 59 (1968); B.H. Voorhees, Phys. Rev. D, 2, 2119 (1970);W.B. Bonnor Gen. Rel. Grav., 24551 (1992).

[12] R. Adler J. Math. Phys. 15, 727 (1974).

[13] H. Bondi, Proc. R. Soc. London, A281, 39 (1964)

[14] R. Tolman, Phys. Rev., 35, 8751930.

[15] F.I. Cooperstock, R.S. Sarracino and S.S. Bayin S. S., J. Phys. A 14, 181 (1981).

[16] Ø. Grøn, Phys. Rev. D, 31, 2129 (1985).

[17] A. Lightman, W. Press, R. Price and S. Teukolsky, Problem Book in Relativity and Gravitation (Princeton University Press, Princeton) (1975).

[18] L. Herrera, A. Di Prisco, J.L. Hernandez-Pastora and N.O. Santos, Phys.Lett. A, 237113 (1998). 